\title{
LA ENERGÍA COMO PARADIGMA DEL CAMBIO URBANÍSTICO Y AMBIENTAL
}

\author{
Carlos Hernández Pezzi \\ Diputación Provincial de Málaga
}

Artículo Recibido: 03/10/2015

Artículo Aceptado: 03/06/2016

"La utopía está en el horizonte. Camino dos pasos, ella se aleja dos pasos y el horizonte se corre diez pasos más allá. ¿Entonces, para qué sirve la utopía? Para eso sirve, para caminar."

E. Galeano

\section{Resumen}

La presente comunicación a Greencities \& Sostenibilidad 2015 se plantea como un esquema de ideas y propuestas articuladas sobre la nueva relación de energía y ciudad, siguiendo las pautas de las Agendas 21 locales, la Agenda Urbana de Málaga, la Estrategia Europea 2020 y los Objetivos del Milenio de la ONU, trabajando con los argumentos básicos, la información y la bibliografía necesaria para centrar los posicionamientos que están a favor de la hipótesis de que el urbanismo del siglo XXI ha cambiado radicalmente de retos y objetivos. Agenda Urbana de Málaga. Como antesala de la Conferencia Mundial United Nations Conference on Housing and sustainable urban development Habitat III en Quito en octubre de 2016, el Foro Greencities \& Sostenibilidad, en octubre de 2015, es una plataforma de acción y difusión para lanzar la Agenda Urbana de Málaga y proponer cambios en las directrices y orientaciones del urbanismo en España.

Palabras clave: Energía; ambiente; recursos; complejidad; lucha contra el cambio climático; Agenda Urbana de Málaga; igualdad de género; calidad democrática; igualdad de oportunidades; acción intergeneracional; energía social; arquitectura Zero CO2; Ciudades; carbono neutral; reducción global de emisiones; turismo hotelero Cero CO2 


\section{WPSReview International on Sustainable \\ Housing and Urban Renewal (RI-SHUR)}

\section{World Urban Forum WUF7}

En el reciente World Urban Forum WUF7 celebrado en Medellín se concluyó lo siguiente: "Nos hemos sentado juntos 142 países, 42 ministros, 139 alcaldes y 22.000 personas a debatir cómo queremos las ciudades del futuro; para mostrar que la equidad es fundamento de los Derechos Humanos; que la equidad urbana se debe integrar en la Agenda de Desarrollo post 2015; que las ciudades deben ser inclusivas y que es urgente poner en marcha acciones que desemboquen en un desarrollo urbano sostenible; que nos comprometamos a fomentar esta visión de ciudades como oportunidades". Porque la urbanización, señalaron, es una fuerza de modificación de casi todo y ya desde 2008 "la mayoría de problemas de este mundo son urbanos".

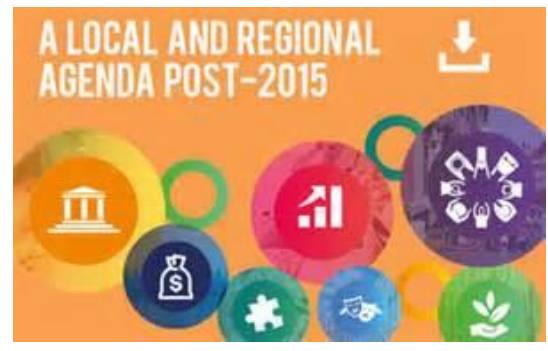

- El marco europeo lo señala la estrategia europea UE 2020 que fija las 5 prioridades siguientes: Empleo, investigación e innovación lucha contra pobreza y exclusión social, educación, cambio climático y sostenibilidad energética.

\begin{tabular}{|c|c|c|}
\hline \multicolumn{3}{|c|}{$\begin{array}{l}\text { Five objectives: } \\
\text { employment, research and innovation, climate change and energy, education, fighting poverty }\end{array}$} \\
\hline $\begin{array}{l}\text { Smart Growth } \\
\text { developing an economy } \\
\text { based on knowledge and } \\
\text { innovation }\end{array}$ & $\begin{array}{l}\text { Sustainable Growth } \\
\text { promoting a more resource } \\
\text { efficient, greener and more } \\
\text { competitive economy }\end{array}$ & $\begin{array}{l}\text { Inclusive Growth } \\
\text { fostering a high-employment } \\
\text { economy delivering social } \\
\text { and territorial cohesion }\end{array}$ \\
\hline \multicolumn{3}{|l|}{ INNOVATION } \\
\hline $\begin{array}{l}\text { Flagship initiative } \\
\text { «Innovation Union" }\end{array}$ & $\begin{array}{l}\text { CLIMATE, ENERGY, } \\
\text { MOBILITY }\end{array}$ & $\begin{array}{l}\text { EMPLOYMENT AND } \\
\text { SKILLS }\end{array}$ \\
\hline $\begin{array}{l}\text { EDUCATION } \\
\text { Flagship initiative } \\
\text { "Youth on the move }\end{array}$ & $\begin{array}{l}\text { Flagship initiative } \\
\text { "Resource efficient Europe" }\end{array}$ & $\begin{array}{l}\text { Flagship initiative } \\
\text { "An agenda for new } \\
\text { skills and jobs" }\end{array}$ \\
\hline $\begin{array}{l}\text { DICIGTAL SOCIETY } \\
\text { Flagship initiative } \\
\text { «A digital agenda for Europe } \%\end{array}$ & $\begin{array}{l}\text { COMPETITIVENESS } \\
\text { Flagship initiative } \\
\text { «An industrial policy for the } \\
\text { globalisation era» }\end{array}$ & $\begin{array}{l}\text { FIGHTING POVERTY } \\
\text { Flagship initiative } \\
\text { "European platform against } \\
\text { poverty" }\end{array}$ \\
\hline
\end{tabular}

WPS RI-SHUR, nํㅜ, 2016, vol.1, ISSN: 2387-1768 


\title{
WPSReview International on Sustainable
}

\author{
Housing and Urban Renewal (RI-SHUR)
}

- Entre los 8 Objetivos del Milenio la ONU sitúa como ODM7 Garantizar la Sostenibilidad del Medio Ambiente. La Declaración del Milenio de las Naciones Unidas, reconoce las terribles circunstancias de la pobreza mundial urbana. Esta articula el compromiso de los Estados que son miembros para mejorar la calidad de vida para al menos 100 millones de habitantes de los asentamientos urbanos con carencias para el año de 2020 (Objetivo 11, Objetivos de Desarrollo del Milenio No. 7). Aunque 100 millones puedan parecer mucho, de cualquier manera, es únicamente el 10 por ciento de la población mundial actual de los asentamientos urbanos con carencias, la cual, no se controla, y se triplicará a 3 billones para el año 2050. El Objetivo 10 se enfoca a la reducción en la mitad de quienes no cuentan con acceso seguro y sustentable de agua potable.

El trabajo de ONU-HABITAT, como el punto clave para la implementación de la Agenda del Hábitat, la Declaración de la Ciudades y otros Asentamientos Humanos en el Nuevo Milenio en el Objetivo 7 de Desarrollo del Milenio. Los Objetivos 10 y 11, han llamado a la agencia y los gobiernos, autoridades locales y regionales, sociedad civil, y al sector privado que está cada vez más cerca a la vida de los pobres que viven en asentamientos urbanos. Aunque los beneficiarios de ONU-HABITAT son personas que viven en la pobreza urbana, su audiencia clave sigue siendo el responsable político en cada nivel con el poder y la autoridad para abordar la pobreza urbana proporcionándoles de recursos, removiendo barreras y asegurando sus derechos civiles y humanos.

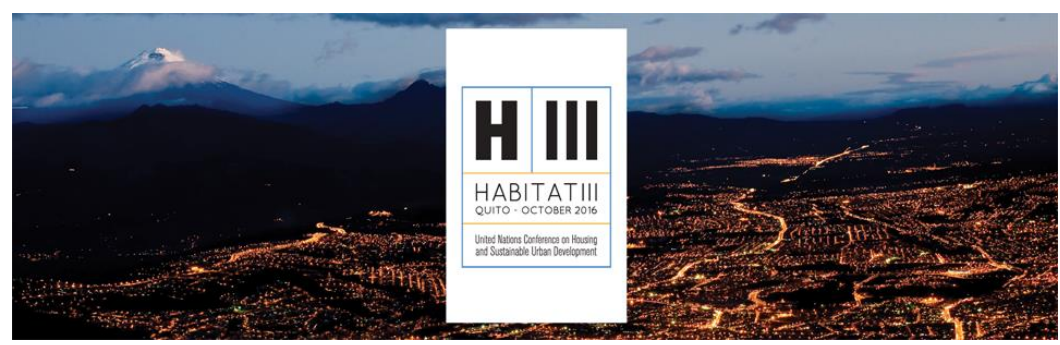

Las políticas urbanas han de tener como eje central a la ciudad sostenible. No hay sostenibilidad sin un balance adecuado de la cuestión de los recursos y los usos de las energías. La gestión de este balance es primordial. El paradigma urbanístico cambia y se orienta hacia la energía como vector, porque esta vertebra las nuevas demandas. Las nuevas alternativas de urbanismo se dirigen a lo que se podría denominar como urbanismo energético: Clima, energía y movilidad eficientes en uso de recursos, como plantea la UE. La estrategia de competitividad se entiende en este marco, que consiste en contraponer a las cuestiones claves soluciones innovadoras que se complementan y se articulan de forma diferente en cada sitio y cooperan y compiten en la medida en que son complementarias. 
WPSReview International on Sustainable

Housing and Urban Renewal (RI-SHUR)

\begin{tabular}{|c|c|c|}
\hline $\begin{array}{l}\text { Smart Growth } \\
\text { developing an economy based on } \\
\text { knowledge and innovation }\end{array}$ & $\begin{array}{l}\text { Sustainable Growth } \\
\text { promoting a more efficient, greener } \\
\text { and competitive economy }\end{array}$ & $\begin{array}{l}\text { Inclusive Growth } \\
\text { fostering a high-employment economy } \\
\text { delivering social andteritorial } \\
\text { cohesion }\end{array}$ \\
\hline $\begin{array}{l}\text { Innovation } \\
\text { "Innovation union" }\end{array}$ & $\begin{array}{l}\text { Climate, energy and mobility } \\
\text { "Resource officient Eurase" }\end{array}$ & $\begin{array}{l}\text { Employment and skills } \\
\text { "An agenda for new skills and jobs" }\end{array}$ \\
\hline $\begin{array}{l}\text { Education } \\
\text { "Youth on the move" } \\
\text { Digital society } \\
\text { "A digital agendo for Eurooe" }\end{array}$ & $\begin{array}{l}\text { Competitiveness } \\
\text { "An industrial policy for the } \\
\text { globalisation era" }\end{array}$ & $\begin{array}{c}\text { Fighting poverty } \\
\text { "European platform against poverty" }\end{array}$ \\
\hline
\end{tabular}

\section{Las grandes cuestiones abarcan todos los recursos y sistemas energéticos combinados.}

- Zero emisiones.

2070

Arquitectura Zero $\mathrm{CO} 2$

- Carbono neutral

Reducción global de emisiones

- Suelo como recurso

Compacidad de la huella urbana

- Agua

Cultura, servicios y ahorro del agua

- Sol y viento

Empleo de energías renovables

- Geotermia, biomasa, otras

Energías limpias complementarias
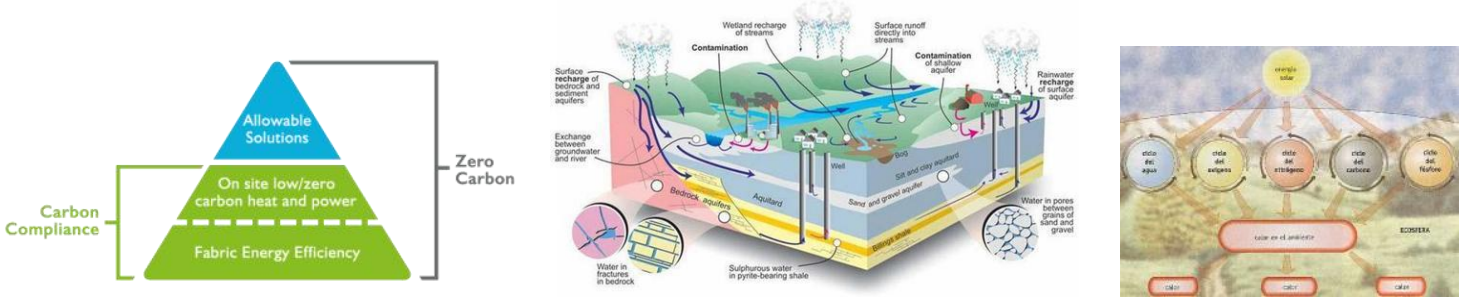

Las políticas urbanas de la ciudad sostenible han cambiado: difieren notablemente de las seguidas hasta ahora. Eso implica cambiar las mentalidades, los instrumentos y las prácticas en un marco colaborativo, interconectado, mezclado y diverso en el que muchas ideas contrapuestas compiten.

Van desde las opciones tecnológicas, soluciones urbanas, alternativas institucionales, programas de mercadotecnia imagen y proyección global, ferial, o de coordinación entre ciudades, a las propuestas de colaboración público-privada, los programas de la UE o la ONU, los de cooperación internacional, los programas de ayuda, las experiencias low energy, etc. 


\title{
WPSReview International on Sustainable
}

\author{
Housing and Urban Renewal (RI-SHUR)
}

Se combinan con programas sociales, educativos, de empleo, de ahorro, de emergencia de ayuda y prevención de catástrofes.

La crisis económica del capitalismo financiero e inmobiliario es la causa de la actual crisis de la ciudad y la vivienda, pero no se suele mencionar más que desde la crítica marxista. Las ciudades emergentes están analizando la ciudad con más acierto que las ciudades europeas, instaladas en el conformismo capitalista y sus derivados simbólicos acerca del neo-liberalismo en la ciudad que pujan por implantar políticas de saqueo de las hipotecas sub-prime y los fondos de inversión de riesgo, que fluctúan entre los mercados de suelo, la energía y la banca internacional. El análisis está incompleto sin estos componentes

La nueva relación entre ciudad y energía necesita responder a nuevas demandas, retos, e instrumentos: No podemos mantener los paradigmas urbanísticos que nos llevaron a la crisis. Tampoco el tipo de planeamiento de ocupación de suelo que se consolidó desde 1998 (todo suelo es urbanizable) y 2007 cuando se promulgó la ley 8/2007 y el Texto Refundido 2/2008 de la ley del suelo de 20 de junio de 2008. Nuestro marco más reciente no se ha demostrado eficaz para detener la destrucción/transformación/hipoteca del suelo y atender a los nuevos objetivos de la planificación urbana y territorial. Los planes se utilizan para fijar marcos administrativos, competenciales y burocráticos, pero las estrategias de ciudad y sus demandas de sostenibilidad y energía se suelen dejar a la planificación sectorial o a planes especiales. Predominan los PGOU, POTs, Parciales y Estudios de Detalle frente a los planes de recursos y optimización que se hacen a posteriori

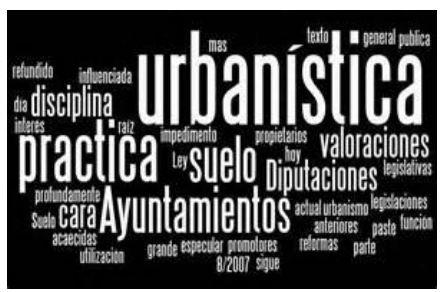

El Estado autonómico y la tradición urbanística española adolecen, entre otros problemas, de bulimia legislativa urbanística; concurrencia de competencias administrativas sin jerarquías claras; atraso y falta de prospectiva en planes y programas relativos a territorios pertenecientes a varias comunidades; una burocracia que propicia la corrupción y la falta de transparencia, y una propensión a la ocupación indiscriminada de lugares de alto valor natural (a veces por los propios gobiernos regionales), a la asfixia de recursos energéticos y al alto consumo de suelo y agua. Frente a esta invasión del territorio los retos urbanos y territoriales implican una nueva visión, nuevas herramientas, nuevos planes (no sólo territoriales, generales, parciales, etc.), sino especiales de rehabilitación, de impacto, de energía, de cooperación,

WPS RI-SHUR, nํㅜ, 2016, vol.1, ISSN: 2387-1768 


\title{
WPSReview International on Sustainable
}

\author{
Housing and Urban Renewal (RI-SHUR)
}

mixtos, estratégicos, etc. Los Planes Especiales, Planes de Protección, Planes de Eficiencia Energética, de Rehabilitación Energética, de Rehabilitación funcional (Ascensores, ITEs, etc.)

De electrificación, suministro o depuración de aguas, de arbolado, contra islas de calor, de movilidad, etc., parecen tener jerarquías menores y secuencias temporales más dilatadas en el tiempo. Esta situación afecta a la subordinación existente hacia las cuestiones siguientes.

- Rehabilitación energética de edificios

- Movilidad sostenible

- Ecobarrios, rehabilitación energética de distritos

- Planificación estratégica de recursos energéticos

- Políticas urbanas sostenibles

- Indicadores urbanos de sostenibilidad

- Rehabilitación de recursos, redes y servicios de infraestructura
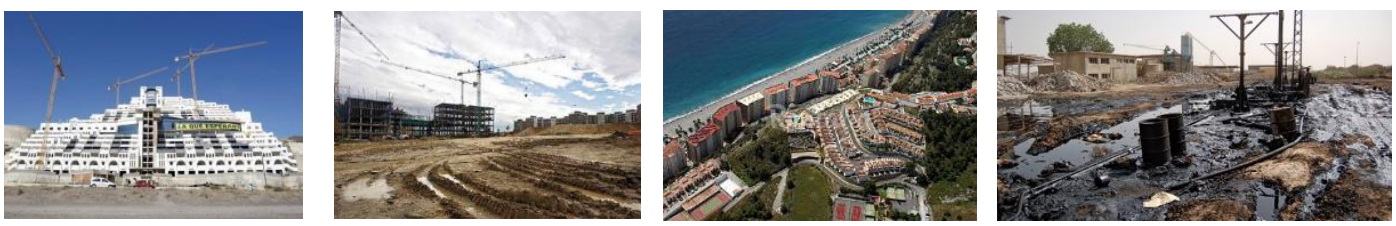

Las imágenes hablan por si solas: simbolizan la realidad del crecimiento. El cambio hacia un urbanismo sostenible está por hacer: Será largo y complejo el proceso cultural hacia un urbanismo responsable con el medio ambiente y la energía, más aún con la regulación de suelo actual y con la mentalidad desarrollista imperante en España. Entre tanto, asistimos a una disociación entre el urbanismo administrativo real y el urbanismo retórico figurado, muchas veces virtual.

Además de la obsolescencia del urbanismo de asignación de usos e intensidades solo, se ha avanzado poco en el urbanismo de la rehabilitación, la sostenibilidad y la energía. No se han elaborado tampoco planes de compacidad y reducción de la huella urbana. El marco urbanístico es el mismo anterior a la burbuja.

Las políticas de vivienda nueva han buscado siempre el urbanismo de asignación de derechos de propiedad y de promoción residencial nueva, por lo que ahora mantienen hipotecado un gran patrimonio privado en expectativa de venta, pero ya clasificado y calificado, lo que ha dado lugar a la denuncia de numerosos convenios urbanísticos imposibles de cumplir; muchos de los suelos pertenecen a instituciones bancarias. Seguimos con el mismo planeamiento pasivo y basando toda la estrategia en la vivienda nueva frente al urbanismo energético y la rehabilitación. 


\title{
WPSReview International on Sustainable
}

\author{
Housing and Urban Renewal (RI-SHUR)
}

El cambio en las políticas de vivienda (Rodríguez López 2015) ${ }^{1}$ en 2015 no ha encontrado alternativas ni cambios de importancia en materia urbanística.

Sobre ciudades sostenibles: Dualidades de uso común. La delgada línea roja entre estrategias de mercadotecnia, las ferias, los lobbies, las marcas de ciudad, las estrategias globales de grandes compañías y las ciudades innovadoras en múltiples aspectos esenciales para los ciudadanos conviven y se agolpan en acontecimientos indiferenciados, ferias, congresos y exposiciones. En estos eventos, múltiples estrategias se solapan hasta hacer irreconocibles los orígenes y los destinos de cada proceso urbano, sus planes generales de ordenación convencionales en relación con las propuestas de moda.

Las tácticas de confluencia de economías de ciudad a distintas escalas regionales, competitividades urbanas subordinadas, procedimientos de publicidad 0 comercialización, se superponen a esferas de sostenibilidad asociables a cada propuesta tecnológica de las grandes compañías de comunicación, transporte o energía. Así, entre competitividad y cooperación se están desarrollando encuentros de todo tipo que se confunden con turismos de congresos, encuentros académicos, cooperación entre ciudades y aplicación de herramientas de domótica, informacionales o de eficacia en telecomunicaciones y energética, tanto a escala de distrito, urbana como metropolitana.

La mercadotecnia se ha instalado en las ciudades como una variable más de su proyección internacional, pero no puede ser la principal, ni la excusa para marginar a las Agendas 21 Locales a favor de las otras variables de gestión y gobierno, de gestión estratégica, de ciudad inteligente o de creatividad y marca, muy sujetas a baremos y datos competitivos de tipo macro. Son los principios cooperativos de las redes de ciudades los que contribuyen mejor al cambio en la ciudadanía y su calidad de vida.

1RODRIGUEZ LÓPEZ, Julio. Marzo 2015 http://socialurban.blogspot.com/2015/03/la-politica-de-vivienda-debe-reaparecer.htm/\#!

RESUMEN: Hasta 2014 han tenido lugar cambios sustanciales en la política de vivienda en España, entre los que destacan. Primero: Endurecimiento del crédito bancario, sobre todo del crédito a promotor. Ello implica más dificultades para acceder a un préstamo bancario para la compra de la vivienda, en especial para los hogares de bajos ingresos. Sobre todo implica la desaparición del supuesto de las VPO, esto es, la financiación a promotor, público y privado, a largo plazo en buenas condiciones de tipo de interés y plazo. Segundo: es evidente la reestructuración de la demanda de vivienda en favor del alquiler, como consecuencia de los nuevos empleos, inestables y con salarios reducidos (las estadísticas del mercado de alquiler en España se limitan solo a los precios de alquiler). Las condiciones de los nuevos empleos complican el acceso a la vivienda en propiedad. En la fuerte tendencia hacia el alquiler ha podido influir, además, el mayor conocimiento de no poder hacer frente al pago de un crédito hipotecario. Tercero: La cuestión de los desahucios ha pasado a ocupar el primer plano en la problemática de la posibilidad de disfrute de la vivienda en España. Habría que tener en cuenta que, junto a los desahucios derivados de la ejecución de una hipoteca para compra de la vivienda habitual, hay también un número importante de desahucios derivado del impago del alquiler. La suma de los desahuciados por ambos conceptos superó los 60.000 anuales en 2013-2014 (Grafico 2).Cuarto: Se han revisado a la baja las previsiones de demanda de vivienda derivada de la creación de nuevos hogares. La recientemente publicada estadística de hogares del INE revela que, frente a los 424.769 nuevos hogares que se crearon en España entre 2002 y 2007, dicha creación bajó hasta 264.140 entre 2007 y 2014. En dicha estadística se ha previsto un ritmo medio anual de 67.000 nuevos hogares para el periodo 2015-2018.

La previsión citada implica menores necesidades de nuevas viviendas que en la etapa previa a la burbuja. Se deben de construir nuevas viviendas, pero el ritmo de nueva construcción debe de estar en línea con las previsiones de demanda. Tras lo sucedido en los últimos años, ha debido de quedar claro que los excesos en la promoción inmobiliaria los pagan todos los españoles, no solo los promotores y los ayuntamientos que permiten tales excesos. 


\section{WPSReview International on Sustainable \\ Housing and Urban Renewal (RI-SHUR)}

La mercadotecnia tiene sentido en el logro de desafíos globales si da cuenta demostrada de resultados y evaluaciones reales en los social, ambiental y energético tanto como en otras materias menos o nada tangibles.

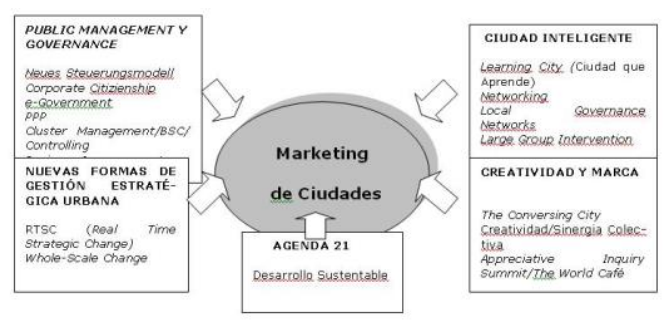

En todo el mundo, los casos de análisis también se compaginan con el autobombo de ciudades, innovadoras, emergentes, resilientes, verdes, "en movimiento", saludables, culturales, etc. Un ejemplo, "La cita más importante en Latinoamérica sobre ciudades inteligentes que reúne a las principales instituciones y personalidades que lideran el cambio y la transformación urbana" se denomina URBAN INNOVATION TOWARDS EQUITABLE CITIES In Latin America y contempla estos grandes asuntos: Ciudades sostenibles; Ciudades equitativas; Tecnología y emprendimiento; Gobierno abierto; Ciudades vivas.

smart city expo PUEBLA

Los casos particulares de ciudades con etiquetas, (labels, brandings o marketings) diferenciados, se enfatizan por la presión medática junto con los casos de éxito generalizables o globales. Es significativo el ejemplo de Chicago que se publicita a partir de su clima característico como "the windy city"; Medellín, proclamada como "ciudad más innovadora del mundo". Especialmente llamativos los casos de las ciudades de las riberas del mediterráneo y su puja por defender modelos propios.

Hay muchos elementos transversales de las ciudades en red que trabajan en común y ponen los paradigmas de enlace por encima y por delante de marcas y ránkings, que muchas veces responden solo a las identidades competitivas y a las soluciones tecnológicas o informacionales. La "marca ciudades" debe ir sustituyendo paulatinamente a los modelos de "marca ciudad" de éxito aislado, que sólo actúa sobre un acontecimiento o proceso excepcional, como pueden ser los juegos olímpicos y los impactos de transformación excepcionales. 


\title{
WPSReview International on Sustainable
}

\author{
Housing and Urban Renewal (RI-SHUR)
}

Resulta paradójico que las grandes ciudades ocupen puestos de relevancia en las escalas de competitividad local cuando son las ciudades medias e intermedias las que menor huella ecológica consumen, suelen ser más compactas y favorecen usos racionales de la energía. En ese empeño está la ONU y numerosos organismos internacionales.

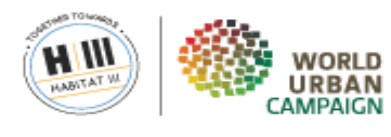

Ciudades emergentes y Ciudades sostenibles: son dos de los elementos puestos en común porque globalizan problemas complejos y propuestas alternativas sobre energía. La dicotomía entre realidades y ficciones es consustancial al mundo contemporáneo. La realidad virtual como soporte de imagen, comunicación y trampantojo es un hecho multiplicado por las tecnologías TICs. La utopía de la ciudad sostenible y el mito de la ciudad inteligente, a veces se contradicen con las prácticas colaborativas, soluciones mixtas e híbridas de los distintos agentes, co-partenariados y tipos de financiación y gobiernos locales.

Frente a la ficción y la tech-fiction hay que avanzar sobre modelos realistas, evaluando y verificando los indicadores y utilizando la prospectiva para adelantarse a los procesos anticipando los impactos, desvíos y fenómenos colaterales que introduzcan variables en las ecuaciones de energía y uso de recursos

La metodología del modelo del Banco Interamericano de Desarrollo (BID), aplicada a las distintas variables regionales y climáticas específicas de cada país es de los más interesantes, pero no entra tanto en aspectos cruciales que se contienen en los de la UE, las Agendas 21 Locales y los objetivos del milenio de ONU Hábitat. La Agenda cultural entra de lleno en ese cambio de perspectivas.

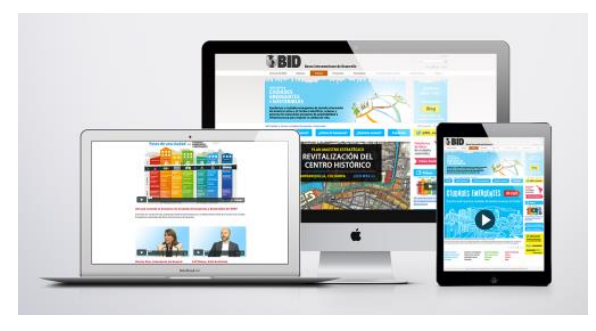

En muchos modelos vigentes se incide demasiado en el monitoreo, el seguimiento, la participación y menos en los controles democráticos de los procesos de toma de decisiones y prioridades, las apuestas de mayor impacto social, el cogobierno o gobernanza de la ciudadanía y la protección de las poblaciones más vulnerables

WPS RI-SHUR, nำ, 2016, vol.1, ISSN: 2387-1768 


\section{WPSReview International on Sustainable \\ Housing and Urban Renewal (RI-SHUR)}

debido a procesos de exclusión. Los indicadores de sostenibilidad urbana deben tener una función de anticipación prospectiva y no de mero observatorio de seguimiento, los planeamientos deben adelantarse a los proyectos y no ir a la zaga.

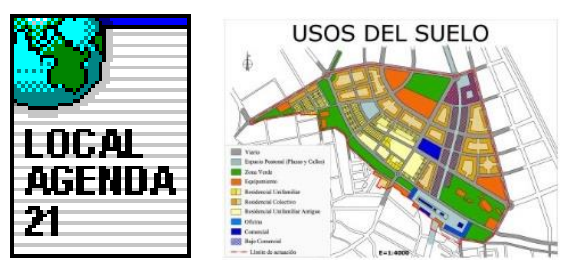

\section{Objetivar el equilibrio social y ambiental de la energía en la ciudad sostenible}

- Las dicotomías entre ciudades virtuales y reales no valen como coartadas de la ciudad inteligente o innovadora. Son meros símbolos de los proyectos estratégicos.

- Los planes urbanísticos deben pasar a contemplar energía y sostenibilidad y políticas de rehabilitación en primer plano.

- La sostenibilidad y la innovación de la noción de ciudad socialmente inteligente, no pueden disociarse, están íntimamente unidos

- La desigualdad y la pobreza no son sostenibles. La lucha contra la desigualdad y la pobreza es una de las primeras aspiraciones de la ciudad sostenible; acabar con la pobreza y la desigualdad en el uso de recursos naturales y energéticos es prioritario

- La variedad de situaciones y climas obliga a manejar y gestionar Instrumentos Diversos: I+D+l; A21Locales; UE 2020; ONUH; BID. Obsérvese la cantidad de instituciones que luchan por objetivos similares. Desde la London School of Economics (LSE), al emtech MIT o a las redes de ciudades

- Las estrategias de mercadotecnia han de combinarse con las exigencias de equilibrio y responsabilidad en los límites del crecimiento, en los proyectos y en los planes. No puede suplantar la acción conjunta.

- Para asegurar el bienestar ambiental y socio-económico de las generaciones siguientes es preciso ahorrar energías y generar comunidades también compactas, unidas, equilibradas. 


\section{WPSReview International on Sustainable}

\section{Housing and Urban Renewal (RI-SHUR)}

- Energía y recursos deben ser evaluadas continuamente para que estén bajo los umbrales del crecimiento responsable y justo.

- Son sostenibles las ciudades que mantienen sus recursos preservados a medio y largo plazo, siguiendo una estrategia intergeneracional para el uso de los recursos.

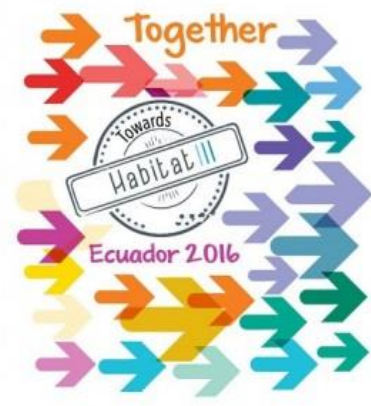

\section{La aplicación al caso de Málaga: Agenda Urbana de Málaga}

El resumen de la Agenda Local 212015 de Málaga, aprobada el 6 de marzo 2015 apunta un considerable $\mathrm{n}^{\circ}$ de propuestas en el sentido de mejorar la cohesión territorial, social, el desarrollo económico, la accesibilidad y el metabolismo urbano, que son característicos del modo de plantear la sostenibilidad en el mencionado documento.

La acción pública resultante es un asunto transversal, que no se basa en la descentralización y la cercanía sino en activas políticas redistributivas precisas sobre cada uso, fiscalidad, equipamiento localización y comunidad. Además, la situación de crisis y la desigualdad territorial en Málaga requieren de instrumentos contundentes y acciones eficientes y contrastables en los plazos que fija la UE para la Estrategia 2020 y el Ayuntamiento de Málaga para las necesidades de la ciudad.

La aprobación de la A21 2015 es tan oportuna como que se complete con una Agenda Urbana en línea con la ONU y su próxima conferencia del Hábitat 2016.

Principales medidas de la Agenda Urbana de Málaga:

1. Elaborar un Plan cuatrienal de Deuda Cero.

2. Mejorar la eficiencia urbana para conseguir acercar a Málaga al objetivo de Cero desahucios

3. Mejorar la eficiencia urbana para conseguir acercar a Málaga al objetivo de Cero emisiones en edificios públicos y ampliación de arquitecturas CeroCO2

4. Mejorar la eficiencia urbana para conseguir acercar a Málaga al objetivo Cero Pobreza Urbana, energética, infantil, alimentaria 


\title{
WPSReview International on Sustainable
}

\author{
Housing and Urban Renewal (RI-SHUR)
}

5. Mejorar la eficiencia urbana para conseguir acercar a Málaga al objetivo de Pleno empleo con garantías de estabilidad, seguridad, dignidad y diversidad. Empleo Verde.

6. Plan de Reequilibrio presupuestario de barrios consignado en los Presupuestos de 2015-2019

7. Plan de preservación del patrimonio municipal, suelos públicos y promociones públicas de vivienda Social. Ordenanza de CeroCO2 en instalaciones hoteleras

8. Plan de Abaratamiento, Mejora y Accesibilidad de los Servicios públicos y de los recursos energéticos.

9. Plan Especial Málaga Educa en Sostenibilidad Turística y Ambiental.

10. Plan de Impacto Social en las principales inversiones municipales dentro del Plan-Marco de Igualdad de Oportunidades de la Ciudad de Málaga.

\section{Grandes cuestiones}

Del enjambre de problemas y alternativas se han extraído unas pocas de diferente signo. La bibliografía y los enlaces dan somera cuenta de ello, incluyendo los organismos internacionales, el análisis de marcas, los entes desde la LSE al MIT, o las referencias a modelos discutidos de las grandes ciudades competitivas como Londres y Barcelona, cuyos modelos padecen también períodos de auge y decadencia.

En los actuales modelos mayoritarios de las redes de ciudades habría que enfatizar y priorizar por encima de los meros análisis de los indicadores, de las marcas y las estrategias de mercado. Eso ya lo hacen los economistas de los fondos de inversión.
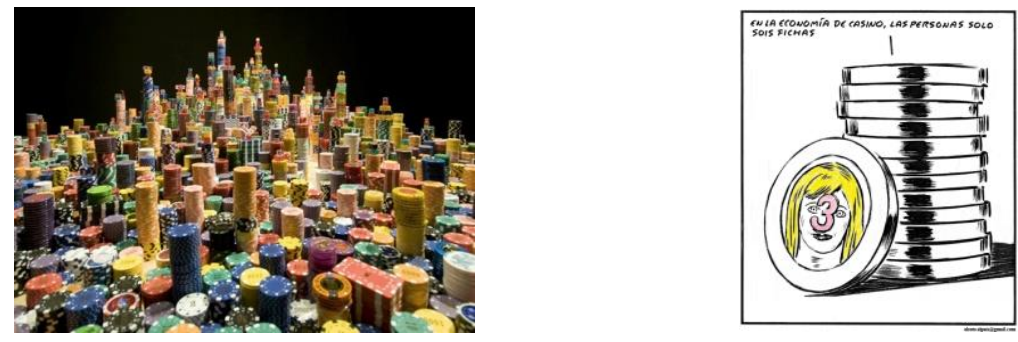

Además de la observación de los procesos de seguimiento y monitorización, de las soluciones tecnológicas y de los despliegues de TICs han de estudiarse a fondo las cuestiones esenciales. A menudo, los análisis de los procedimientos prospectivos de mayor impacto se pierden en las soluciones técnicas e instrumentales, en los contadores de suministros y redes, en la información inteligente y pormenorizada de ahorros y disponibilidades, etc., y no en las cuestiones básicas de mejora, ahorro y yacimientos de nuevos recursos energéticos.

Fruto de la visión competitiva y cuantitativa que se está ofreciendo de los retos de las ciudades estamos dejando a un lado los objetivos centrales que perseguimos en el uso, acceso, consumo y renovación, diversificación y democratización (precios, tasas, universos de población) de las energías contempladas sino como derechos urbanos a

WPS RI-SHUR, nํ, 2016, vol.1, ISSN: 2387-1768 


\title{
WPSReview International on Sustainable
}

\author{
Housing and Urban Renewal (RI-SHUR)
}

los servicios y redes de la calidad de vida. En el agua y la electricidad se están produciendo movimientos importantes y concentraciones de capital y compañías que no deben ir en beneficio de un modelo caducado sino de nuevos modelos de apropiación de la ciudad sostenible.

Estos elementos de un nuevo urbanismo energético para revisar la visión del territorio como mero suelo a colonizar, son los que hay que incluir en visiones integrales; entre otros: Energías diversificadas, ambiente, recursos, complejidad. Lucha contra el cambio climático. Igualdad de género. Calidad democrática. Igualdad de oportunidades. Acción intergeneracional. Inteligencia ciudadana. Energía social. Al menos estos 6 objetivos son cruciales:

- Nuevas políticas de rehabilitación energética y de rehabilitación funcional de viviendas

- Nuevas políticas de alquiler frente a obra nueva

- Nuevas políticas urbanas de planificación de la energía y recursos

- Nuevas políticas de servicios urbanos y movilidad sostenible

- Nuevo impulso contra el cambio climático, islas de calor, contaminación, residuos

- Nuevos planes de urbanismo energético, resiliencia, prevención y emergencia

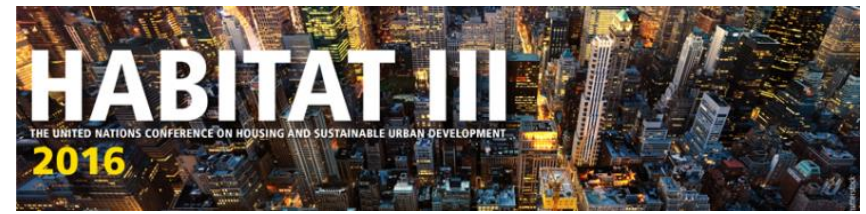

La innovación en el reparto y distribución de la tecnología compartida y comunitaria será un reto de las energías que hay que aportar a las ciudades en el presente siglo. Una ocasión inmejorable será la Conferencia Mundial del Hábitat a celebrar por la ONU en Quito (Ecuador) en 2016. La descentralización de la innovación, el papel de las nuevas ciudades de la innovación y la sostenibilidad entendida como un cambio de paradigma del urbanismo al medio ambiente serán los principales factores de las nuevas estrategias, de los liderazgos locales fuertes y de las mejoras de las brechas de desigualdad.

El urbanismo de la energía es un primer paso para focalizar un cambio de actitud ante los problemas urbanos que ya es inexorable, tanto en las políticas comunes de la humanidad contra el cambio climático, como acerca del nuevo papel que han de jugar las ciudades medias e intermedias, las más sostenibles en nuevos modelos de ocupación, crecimiento y bienestar de la población urbana.

ONU Hábitat reclama una Nueva Agenda Urbana que ponga mayor precisión en las políticas mundiales y los retos de las ciudades. Gran parte de esa agenda será la tarea prioritaria de las ciudades pioneras en los cambios urbanos y de las redes que ellas mismas formen para compartir sus fuentes, repartir sus recursos, diversificar los

WPS RI-SHUR, n3, 2016, vol.1, ISSN: 2387-1768 


\title{
WPSReview International on Sustainable
}

\author{
Housing and Urban Renewal (RI-SHUR)
}

riesgos y ser más resilientes y solidarias a las crisis económicas, ambientales y a las catástrofes sobrevenidas de la naturaleza o de los desastres de la avaricia capitalista de este período de casino sin reglas. Hay que definir la Agenda Urbana 2016 de nuestras ciudades y aportarla a la hoja de ruta anterior y posterior a la United Nations Conference on Housing and sustainable urban development Habitat III en Quito en octubre de 2016

\section{Bibliografía. LINKS}

\section{Ley del suelo 7/2008}

http://www.boe.es/boe/dias/2008/06/26/pdfs/A28482-28504.pdf

\section{Hernández Pezzi, Carlos}

Hernández Pezzi, Carlos. "Crecer o madurar. El modelo de urbanismo energético" Modelos de crecimiento urbano: XV Seminario sobre Gestión Pública Local, (15. 2009. Gijón). Editorial Trea. 2010 ISBN 978-84-9704-505-6, págs. 18-30.

Hernández Pezzi, Carlos. "El urbanismo en la era de la crisis". Congreso Sustainable Building 2010 SB10mad

http://www.sb10mad.com/ponencias/archivos/d/D008.pdf

\section{Rodríguez López, Julio}

http://socialurban.blogspot.com/2015/03/la-politica-de-vivienda-debe-reaparecer.htm|\#!

\section{ONU Hábitat. Clos, Joan}

http://comunicacion.senado.gob.mx/index.php/informacion/versiones/14808-

conferencia-magistral-del-senorioan-clos-director-ejecutivo-de-onu-habitat.html

...."En la Conferencia de Naciones Unidas sobre los Asentamientos Humanos, la última en 1996 en Estambul, se adoptaron compromisos para lograr dos objetivos:...Veinte años después, en 2016, tendremos la Tercera Conferencia sobre Vivienda y Desarrollo Urbano Sustentable, Hábitat III, acordará cómo lograr que el desarrollo urbano sostenible en el marco de la Agenda de Desarrollo, pueda entonces renovar y redireccionar las líneas de acción de 1996,...como respuesta a uno de los más importantes acuerdos aprobados en la Conferencia Río+20. En 2010, Naciones Unidas echó a andar un proceso de negociación; ya tenemos 17 objetivos; nótese que hoy en día los Objetivos del Milenio son sólo 8, ya tenemos 17 objetivos y 169 metas que van a servir como insumo para la negociación...A diferencia de 2000, la Agenda de Desarrollo Post 2015, va a ser universal y va a tener indicadores; y recogerá todas las lecciones aprendidas de estos últimos 15 años y donde, desde luego, habremos de incorporar temas y metas que no figuraban en la agenda de 2000."

\section{Ciudades emergentes y sostenibles}

http://www.monzamora.com/22351/3862820/-architecture-photographydesign/infografias-metodologia-ciudades-emergentes

http://blogs.iadb.org/ciudadessostenibles/2015/04/07/movilidad-urbana/ 
WPSReview International on Sustainable

Housing and Urban Renewal (RI-SHUR)

http://www.zerocarbonhub.org/event/16

http://grist.org/cities/carbon-zero-chapter-1-cities-in-the-age-of-climate-consequences/

http://www.technologyreview.com/emtech/digital/15/

LSE Cities

http://files.Isecities.net/files/2014/11/LSE-Cities-2014-The-Transformative-Role-ofGlobal-Urban-Growth-NCE-Paper-01.pdf

\section{LSE Cities}

Mohsen Mostafavi is the Dean of the Harvard University Graduate School of Design and the Alexander and Victoria Wiley Professor of Design.

http://secities.net/media/objects/events/2011-03-15-ecological-urbanism

\section{LSE Cities}

http://www.Ise.ac.uk/publicEvents/LSEWorks.aspx

\section{Transcripción de MARCA CIUDAD: LONDRES}

AA.VV. MARCA CIUDAD LONDRES ¿POR QUÉ LONDRES? JUEGOS OLÍMPICOS 2012 CONCLUSIONES

http://www.creativereview.co.uk/cr-blog/2009/september/oh-london

http://popsop.com/48091

http://www.londonandpartners.com/about-us/business-strategy

http://www.londonandpartners.com

http://ideas.ie.edu/revista-99/londres-gestion-de-la-marca-ciudad-340405/

http://johnsonbanks.co.uk/identity-and-branding/blue-chip/think-london/

LONDON Enterprise panel. Jobs and growth plan for London. Greater London Authority, abril de 2013 http://www.londres.es/historia

Un reto para nuestra profesión, gestionar la comunicación de la marca ciudad y la marca país. En:

http://citybrand.me/tag/marca-pais-2/

http://www.ieee.es/Galerias/fichero/docs opinion/2012/DIEEEO48-

2012_JJOO_2012PlaneamientoEstrategicoyCooperacionInternacional_DeMiguel.pdf

http://gestion.pe/2012/08/01/tendencias/verdadero-triunfo-londres-juegos-olimpicos$\underline{2012-2008887}$

London \& Partners es la organización oficial, sin ánimo de lucro, que se encarga de promocionar a Londres con una sola voz, visión y misión para las audiencias de todo el mundo, trabajando de la mano con el Alcalde de Londres y una red de socios comerciales. OBJETIVOS QUE SE QUIEREN LOGRAR Posicionar Londres como ciudad líder en el mundo. Incrementar estrategias para su desarrollo económico Conservar su posicionamiento en el mundo gracias a los logros alcanzados en los últimos años. Impulsar a las empresas y a los grupos de interés para que puedan

WPS RI-SHUR, no3, 2016, vol.1, ISSN: 2387-1768 


\section{WPSReview International on Sustainable}

\section{Housing and Urban Renewal (RI-SHUR)}

apoyar la promoción internacional de Londres. Ayudar a construir su reputación global antes, durante y después de los Juegos Olímpicos y Paralímpicos. El City Marketing: de teoría a realidad y moda Enfoque interdisciplinario entre: marketing, geografía, arquitectura, sociología, gestión corporativa y gestión pública Se abordan estudios como identidad e imagen, estrategias de marca para productos y servicios, pero esta vez aplicados a ciudad. Empresa Emprendedora=Ciudad Emprendedora El marketing de ciudad pasa a ser más que una herramienta, a ser una filosofía de gestión urbana ¿Cómo se crea una marca ciudad? Dimensión histórica: Identidad Proyección de identidad en forma de imagen. Diseño material de la marca MARCA CIUDAD: BARCELONA MARCA CIUDAD: NY "Lo que hace el neoliberalismo: ya no se habla de Estados sino de 'marca país.", Los comunicadores y la marca ciudad 